\title{
APRENDIZAJE SERVICIO UNIVERSITARIO. EXPERIENCIA EN UNA COMUNIDAD NATIVA AMAZÓNICA PERUANA
}

\author{
UNIVERSITY SERVICE LEARNING. AN EXPERIENCE IN A PERUVIAN \\ AMAZON NATIVE COMMUNITY
}

\author{
Ignacio García Ferrandis, Javier García Gómez \\ Universidad de Valencia
}

\begin{abstract}
Correspondencia: Ignacio García Ferrandis
Correo: ignacio.garcia-ferrandis@uv.es

Recibido: 28/01/20; Aceptado: 09/06/2020

DOI: $10.17398 / 0213-9529.40 .1 .61$
\end{abstract}

\section{RESUMEN}

Este artículo recoge la experiencia de Aprendizaje Servicio (ApS) que se realizó con universitarios españoles y peruanos, mediante acciones de cooperación en una comunidad nativa kechwa de la selva alta amazónica en Perú. Para ello se partió del convenio de colaboración existente entre la Universidad Nacional de San Martin de Perú y la Universidad de Valencia (España), dentro del cual se han realizado el servicio en la Comunidad de Chirikyacu en la región de San Martin (Perú). Las actuaciones del alumnado se realizaron en el periodo de prácticas de ambas instituciones universitarias. Por un lado, las alumnas españolas se integraron en la comunidad para realizar tareas de cooperación, que propiciaron mejoras en la convivencia y en el nivel educativo. Por su parte, el alumnado peruano se centró en la instalación de un huerto comunitario, una granja avícola y en cuestiones relacionadas con la educación para la salud.

Palabras clave: Aprendizaje Servicio; Cooperación; Universidad

\begin{abstract}
This article gathers the experience of Learning Service (LS) that was carried out with Spanish and Peruvian university students, through cooperation actions in a kechwa native community of the high Amazon rainforest in Peru. This collaboration could be carried out thanks to the formal agreement between the National University of San Martin of Peru and the University of Valencia (Spain), within which the service was carried out in the Chirikyacu Community in the San Martin region (Peru). The students' performances were carried out during the internship period of both university institutions. On the one hand, Spanish students were integrated into the community to carry out cooperation tasks, which led to improvements in coexistence between community members and in the educational level. For its part, Peruvian students focused on the installation of a community orchad, a poultry farm and issues related to health education.
\end{abstract}

Keyword: Service Learning; Cooperation; University. 


\section{INTRODUCCIÓN Y ANTECEDENTES}

El concepto de Aprendizaje-Servicio (ApS) viene siendo utilizado desde hace más de cincuenta años, sin embargo, en la actualidad sigue concibiéndose como una propuesta innovadora. Se trata de una metodología actual pero que cuenta con elementos sobradamente conocidos por todos, como son el servicio voluntario y la transmisión de conocimientos y valores. El elemento innovador consiste en vincular estrechamente servicio y aprendizaje en una sola actividad educativa bien articulada y coherente (Puig y Palos, 2006). Como indican Francisco y Moliner, (2010), a pesar de las innumerables definiciones, existe consenso al señalar dos componentes básicos del ApS. Por un lado, el aprendizaje eficaz y de calidad de contenidos conceptuales, procedimentales y actitudinales ligados al currículum. Por otro lado, el servicio y la labor voluntaria que pretende la transformación de la comunidad. En cualquier caso, entendemos el ApS como un método por el cual los estudiantes aprenden a través de la participación en una comunidad para satisfacer las necesidades de la misma. Para ello, se deben coordinar una institución (escuela primaria, secundaria, una institución de educación superior...) y la propia comunidad. El ApS se integra en el currículo académico de los estudiantes, reforzándolo en aquellos componentes educativos del programa de servicio comunitario en el cual los participantes toman parte; además, proporciona un tiempo estructurado para que los estudiantes reflexionen sobre la experiencia del servicio comunitario. Como expone Aramburuzabala (2013) esta práctica educativa, que combina objetivos de aprendizaje curricular con objetivos de servicio a la comunidad, favorece el desarrollo del pensamiento crítico y la conciencia social. De esta manera el ApS desarrolla experiencias transformadoras que convierten a los estudiantes en agentes de cambio con una visión de un mundo más justo.

Este artículo es fruto de las acciones realizadas mediante la metodología de AprendizajeServicio (ApS), durante los últimos 10 años, en la Comunidad nativa de Chyrikyacu (Perú), por estudiantes de la Universidad Nacional de San Martin de Perú (UNSM) y la Universidad de Valencia, España (UV). Durante esos años se han podido desarrollar toda una serie de acciones por dos motivos principalmente. En primer lugar, por la existencia, desde 1996, de un convenio marco de cooperación entre la Universidad Nacional de San Martín (Perú) y la Universidad de Valencia (España) para intercambio académico y desarrollo de proyectos específicos con participación conjunta de docentes y estudiantes de ambas universidades. Por otro lado, cabe destacar las ayudas recibidas en diferentes convocatorias sin las cuales no se podrían haber realizado algunas de las actuaciones que se verán más adelante. A continuación, se describe muy brevemente los proyectos que se llevaron a cabo con las ayudas concedidas.

En Octubre del 2008, la Consellería de Inmigración y Ciudadanía de la Generalitat Valenciana, en la convocatoria de Subvenciones a Proyectos de Cooperación Internacional al Desarrollo (ONGD), aprobó el financiamiento del proyecto "Centro de educación y gestión ambiental para la recuperación y manejo sostenible de los recursos naturales de la microcuenca del alto Cumbaza (Perú)" para ser ejecutado en la Comunidad Nativa Chirikyacu, perteneciente a la provincia de Lamas, Región San Martín, ubicada en la selva alta del Perú. El proyecto fue gestionado por la Fundación de la Universidad de Valencia, haciendo de contraparte la Universidad Nacional de San Martín y de beneficiaria la Comunidad Nativa Chirikyacu. Una vez ejecutadas las acciones programadas en el proyecto, la mencionada comunidad nativa expresó su agradecimiento imponiendo a las instalaciones el nombre de "Valencia wasi" (Casa de Valencia). El objetivo que se perseguía con el proyecto era recuperar ambientalmente la microcuenca y elevar el nivel y calidad de vida de la población, implementando infraestructuras, para diversificar la oferta turística y dotar a la comunidad de alternativas económicamente 
viables. Además de la construcción de un Centro de Educación Ambiental, se mejoraron los caminos de acceso a los lugares atractivos, se capacitó a la población en el manejo, conservación y administración de los mismos y se promovió el turismo organizando visitas guiadas, que aportó beneficios económicos y propició mejorar las condiciones y calidad de vida de la población local.

Terminado el proyecto en 2011 se continuó la labor de cooperación mediante ayudas de la Universidad de Valencia, para mejorar la calidad de vida de la comunidad, desde la sostenibilidad, es decir con un equilibrio entre lo ambiental, sociocultural y económico, atendiendo a los intereses de la comunidad nativa. La Universidad de Valencia convoca periódicamente ayudas para realizar acciones de cooperación y en el año 2014 a través del Vicerrectorado de Internacionalización y Cooperación en la IV Convocatoria Proyectos Cooperación concedió una ayuda al "Proyecto integrado de investigación-acción participativa para el desarrollo sostenible de las comunidades nativas del Alto Cumbaza (Perú)" y en 2016 en la $\vee$ convocatoria, se realizó el "Proyecto Warmiwasi. Centro artesanal para la mujer de Chirikyacu (Perú)". Las intervenciones durante estos años se han centrado en los sectores agropecuario, salud, turístico y ambiental, que a su vez están interrelacionados.

A lo largo de todo este tiempo, los objetivos generales han sido los siguientes:

- Desarrollar en el alumnado competencias básicas como el trabajo en equipos interdisciplinares, el pensamiento crítico, el respeto a la diversidad y el desarrollo de habilidades comunicativas y sociales.

- Promover en el alumnado la participación, la conciencia social, la solidaridad y el compromiso con el entorno.

- Mejorar las condiciones de vida de la comunidad nativa de Chirikyacu.

\section{ACTUACIONES}

El área donde se desarrolla el proyecto se ubica en la parte alta de la microcuenca del río Cumbaza, al Nor-Oeste de la ciudad de Tarapoto y Nor-Este de la ciudad de Lamas, situada en el flanco occidental del Bosque de Protección Regional "Cordillera Escalera", distrito de San Roque de Cumbaza, Provincia de Lamas, Región San Martín. Ecológicamente, es un bosque húmedo subtropical, constituido por terrenos de ladera que están entre los 700 a 1100 m.s.n.m.

En esta zona están asentadas tres comunidades nativas Chunchiwi, Chirikyacu y Aviación, de la etnia lamista y habla kechwa, cuya situación socioeconómica es muy precaria. Los indicadores demográficos y socioeconómicos de las comunidades nativas indican, que la tasa de analfabetismo es de $21.5 \%$, la tasa de desnutrición crónica en niños en edad escolar es de $51.2 \%$, la tasa de mortalidad infantil de 58.5/1000 y un índice de pobreza de $28.4 \%$, que lo ubica en el nivel Muy Pobre (INEI, 2000).

Complementando las acciones llevadas a cabo en los proyectos antes mencionados, se planteó la posibilidad de que el alumnado de la UV y la UNSM realizaran estancias formativas aprovechando las convocatorias de becas de estancias internacionales. En éstas se programaron actividades prácticas contempladas en su curriculum de formación y acciones de cooperación dirigidas a beneficiar a la mencionada comunidad nativa kechwa de la selva alta amazónica del Perú, cumpliendo de este modo con los principios del ApS. Por parte española, participaron alumnas de la Universitat de València, de los grados de Educación Social, Pedagogía, Magisterio, Ingeniería Forestal, Geografía, Trabajo Social y Enfermería o del Máster Universitario en Cooperación al Desarrollo. Siguiendo el espíritu del ApS se integraron en la 
comunidad para realizar tareas de cooperación, y propiciar mejoras en la convivencia y en el nivel educativo. Por su parte, el alumnado peruano pertenecía a titulaciones de Agronomía, Veterinaria y Enfermería, y su actuación se centró en la instalación de un huerto y una granja comunitaria y en aspectos relativos a la educación para la salud.

En la tabla 1 se indica el alumnado español y las titulaciones de los participantes en los distintos años en que se ha desarrollado la actividad.

Tabla 1. Evolución alumnado español participante por año y titulación

\begin{tabular}{lll}
\hline Año & Número de alumnos/as & Titulación \\
\hline 2011 & 1 & Ingeniera Forestal \\
2012 & 3 & Magisterio, Máster de Cooperación, Educación Social \\
2013 & 3 & Magisterio, Educación Social y Enfermería \\
2014 & 1 & Educación Social \\
2015 & 3 & Magisterio, Pedagogía y Educación Social \\
2016 & 1 & Educación Social \\
2017 & 3 & Máster de Cooperación, Pedagogía y Educación Social \\
2018 & 3 & Magisterio y Educación Social \\
2019 & 3 & Magisterio y Educación Social \\
$2020^{*}$ & 3 & Máster de Cooperación, Geografía y Trabajo Social \\
\hline
\end{tabular}

* Participación prevista

Las actividades realizadas estaban relacionadas con los intereses del alumnado en función de su titulación y de los intereses de la población. Por ello cada estancia tuvo acciones diferentes. A continuación recogemos algunas de ellas.

\subsection{Instalación de huerto hortícola}

En colaboración de los estudiantes de la Escuela de Agronomía de la UNSM-T, junto con comuneros interesados de Chirikyacu, se realizó el proyecto de un huerto hortícola (figura 1), donde los estudiantes aprendieron técnicas de siembra y manejo de diversas especies de hortalizas. A su vez, los productos obtenidos se utilizaban para el consumo de las familias de la comunidad a fin de mejorar su dieta alimenticia y también para la alimentación de los visitantes que se alojan en el albergue ubicado en la comunidad.

Los estudiantes participaron en todo el proceso agronómico del huerto, considerándose entre estos, la preparación del terreno, desinfección de semillas, instalación de almácigos, siembra, deshierbes, control de plagas y enfermedades; y cosecha. La realización de estas actividades abarca además acciones de investigación impulsadas desde las materias de edafología, climatología, producción de cultivos y sanidad vegetal, que los docentes comparten con sus estudiantes y los vinculan a la realidad de la comunidad. 


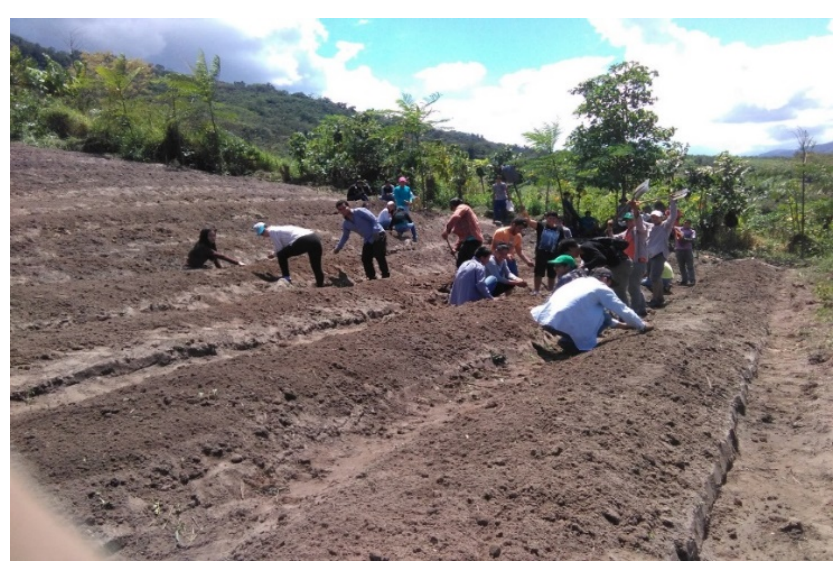

Figura 1: Alumnado en el huerto ${ }^{i}$

\subsection{Granja}

Con el apoyo del alumnado en prácticas y la Comunidad se construyó una granja comunal (figura 2) de $72 \mathrm{~m}^{2}$, para 300 aves con un área de pastoreo circulado, empleando para ello materiales de la zona y siguiendo las indicaciones de coordinación del profesorado de la Escuela de Veterinaria de la Universidad Nacional de San Martín (Perú).

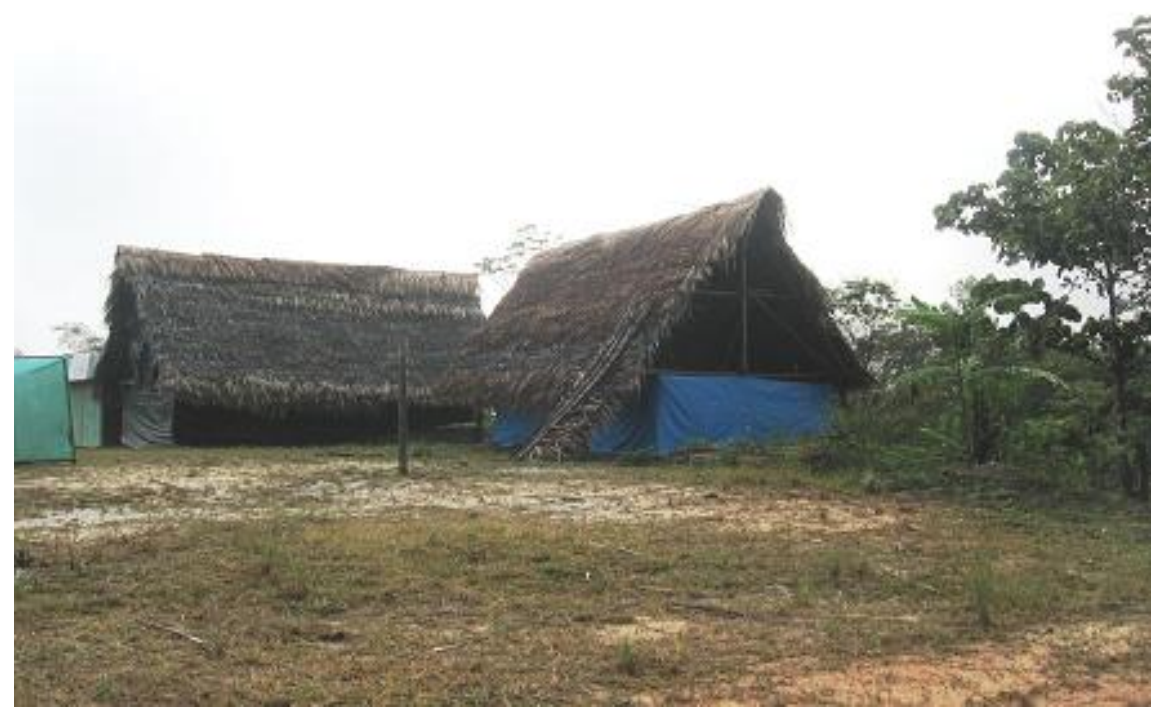

Figura 2: Granja comunal

Se consideró que la mejor alternativa era la crianza de gallinas criollas, adaptadas a la zona, puesto que su manejo es sencillo y los productos que se obtienen son de alta calidad nutritiva y de bajo costo. Con esta actividad se propició el desarrollo social y económico, con la participación de los nativos, como un modelo de desarrollo integral de Comunidades nativas manteniendo su interculturalidad ancestral. Por otro lado permitió disminuir los altos índices de desnutrición complementando la dieta alimenticia con fuentes de proteínas y energéticas; proporcionando huevos y carne con alto valor nutritivo para complementar la dieta familiar nativa de escasos recursos. En el control sanitario participa el alumnado que sigue las normas de bioseguridad con un calendario sanitario establecido. La gestión y manejo de los animales es responsabilidad de cuatro familias, participando padres, madres e hijos conformando un grupo de 12 a 15 personas (figura 3). Cada familia se responsabiliza de la atención a las aves durante una semana, en la que tienen que cuidarlas, proporcionarles el alimento y recoger los huevos. Esta rotación ha hecho que haya un ambiente de mucha compenetración entre los asociados. 
Los socios de la granja avícola participan en la comunidad los domingos con la venta de comidas a los visitantes que acuden a Chirikyacu. La actividad de la granja ha sido reconocida por el FONCODES (Fondo de Compensación de Desarrollo para la zona Rural de la Selva) institución del estado peruano, que premió la iniciativa con la adquisición de la incubadora para 500 huevos de las propias gallinas. Lo que permite multiplicar la productividad con fines de vender pollitos y reproducción de aves de reemplazo para los comuneros de la granja avícola, cerrando así el círculo productivo. En las tareas más específicas como la vacunación participó alumnado de la Escuela de Veterinaria de la Universidad Nacional de San Martín (Perú).

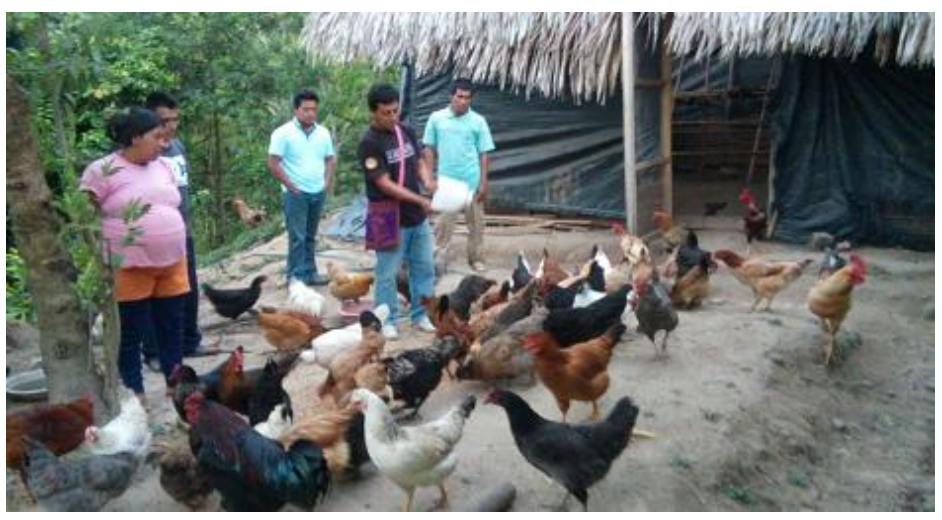

Figura 3: Los comuneros en la granja

\subsection{Construcción del Parque Infantil}

Otro de los objetivos del proyecto era el diseño y construcción de un parque infantil (figura 4) frente a la escuela de la comunidad, en él participaron padres de familia de los niños y niñas del centro educativo. En su construcción se utilizó material de desecho como neumáticos viejos, maderas, clavos, cordeles, pinturas, brochas, etc, que fueron adquiridos por donaciones y pequeños aportes de algunos padres. Una vez terminado el parque infantil, ha sido y es de gran utilidad para el uso y disfrute de los niños y niñas de la comunidad.

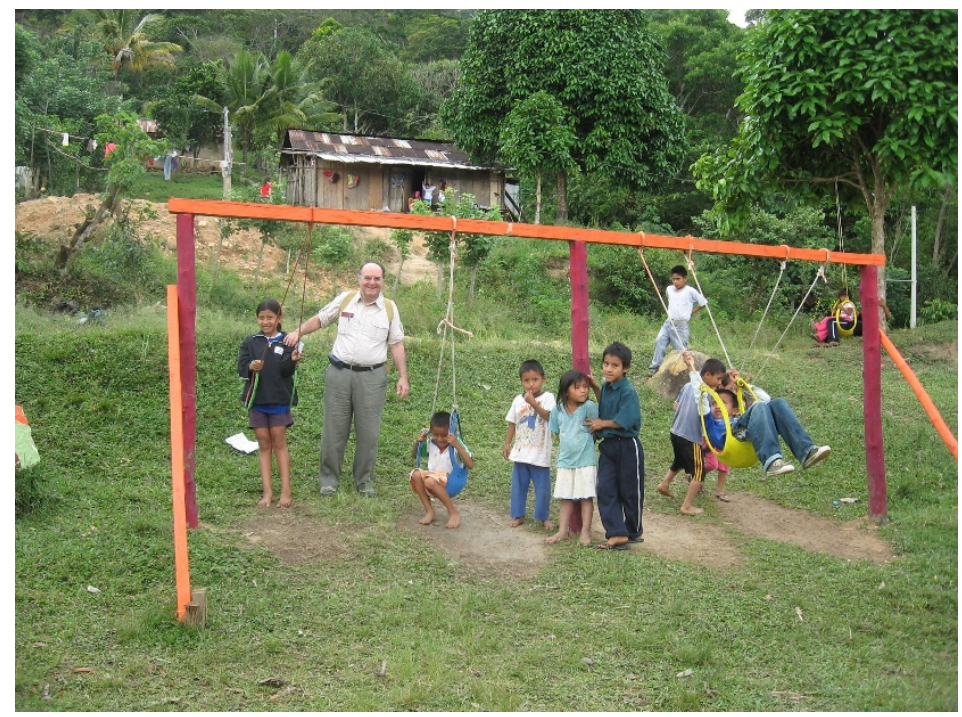

Figura 4: Parque infantil 


\subsection{Apoyo en la construcción de Huaro}

Una estudiante del Máster en Cooperación y de formación arquitecta, participó en la construcción del "Huaro", que es una estructura tipo puente colgante con una canastilla metálica para el traslado de personas de un lado a otro del río. Este se construyó sobre el río Cumbaza con el propósito de facilitar a los comuneros el traslado sin riesgo cuando aumenta su caudal en épocas lluviosas. Bajo la dirección de un Arquitecto docente de la UNSM se ejecutó esta obra, siendo la estudiante de mucho apoyo y a su vez tuvo la oportunidad de aprender "in situ" técnicas de construcción relacionadas con su especialidad.

\subsection{Acciones Formativas}

Dado que mucho del alumnado pertenecía al ámbito educativo (magisterio, educación social, pedagogía), se hicieron actuaciones formativas y divulgativas. En el ámbito de la salud se hicieron talleres sobre nutrición, manipulación, conservación y consumo de alimentos, salud reproductiva, lactancia materna, vacunas, higiene de las manos, para la salud, higiene bucodental, de agua para consumo y aseo personal, vectores y plagas, excretas, aguas residuales y residuos sólidos. Para la capacitación en el manejo turístico se hicieron cursos y talleres sobre recursos y productos turísticos, técnicas gastronómicas, gestión turística y capacitación de guías. En el ámbito ambiental se trabajó el manejo vivero agroforestal y educación ambiental.

\section{Taller de autoestima para mujeres}

Con el propósito de fomentar la autoestima de las mujeres de la comunidad y concienciarlas en la importancia y valor de ser mujer; estudiantes de la Universidad de Valencia, realizaron un taller de autoestima. Este se llevó a cabo mediante un cine-forum, en el cual presentaron la película: Hijas de Belén, incluido dentro de la producción "En el mundo de cada rato". Al final de la proyección se abrió un debate, en la cual participaron las mujeres y varones de la comunidad, resaltando el rol importante de la mujer en la familia.

\section{Educación para la salud}

Se han impartido cursos de nutrición en el que participaron profesorado y alumnado de ambas universidades y entre las actividades realizadas cabe destacar un tratamiento para la cura de pediculosis (figura 5). Se partió de la detección y eliminación de piojos y liendres y así evitar la diseminación e infecciones que originan estos parásitos, y aliviar las manifestaciones físicas y emocionales de la comunidad de Chirikyacu. El tratamiento se hizo tanto en los niños como en los adultos, ya que había un contagio masivo entre todos los habitantes de la comunidad a causa de este parásito. Para el tratamiento se aportaron los productos farmacéuticos necesarios para eliminar estos parásitos. Se aprovechó para divulgar aspectos sanitarios que permita a la población:

- Proporcionar los conocimientos necesarios para detectar y eliminar piojos y liendres, evitando la diseminación.

- Tomar conciencia de la importancia de realizar una revisión periódica para detectar la pediculosis.

- Desarrollar hábitos relacionados con el cuidado de la salud, de la higiene personal y de los otros.

- Establecer medidas de promoción y prevención sobre la pediculosis. 
Participaron los alumnos y alumnas de la Escuela Profesional de Enfermería de la UNSM de Tarapoto, supervisados por profesorado de la misma escuela. También pudo colaborar un alumno del grado de Enfermería de la Universitat de València, España.

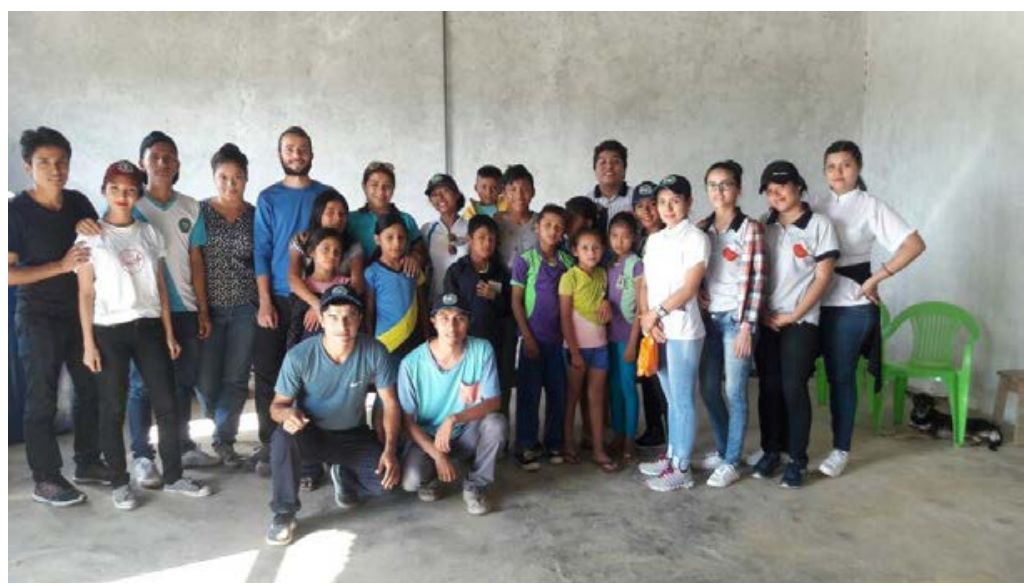

Figura 5: Participantes en el programa de tratamiento de la pediculosis

\subsection{Construcción del warmi wasy (hogar de la mujer)}

El objetivo de esta construcción (figura 6) es dotar a la comunidad femenina de un centro cultural donde realizar actividades educativas, artesanales y lúdicas, así como lugar de encuentro de las mujeres. Con ello se pretende mejorar la cultura de la mujer nativa.

Se planificó mediante un diseño participativo, pretendiendo que las mujeres nativas asuman como propia las instalaciones para un mejor uso de las mismas. La metodología participativa permite desarrollar valores de tolerancia y el respeto hacia el otro y sus ideas, reflejándose sus propuestas en el proyecto. En la reunión inicial se realizó un diagnóstico participativo para clarificar las percepciones que tienen los integrantes de la comunidad donde se plasmaron algunas inquietudes de las mujeres como el uso de material de construcción para el techo a base de hoja de palma, sugiriendo que la casa debe ser un lugar ventilado, con luz natural. Con la ayuda de una arquitecta se logró una propuesta de diseño. Para poder desarrollar la labor y almacenar los productos de artesanía, se construyó un mobiliario para el que se contó con el trabajo del carpintero de la comunidad. Ello contribuyó a generar recursos económicos que repercuten en la mejora de la población. El mobiliario consistió en un armario cerrado para guardar las máquinas de coser, un mueble de estanterías para colocar la producción artesanal, dos mesas de trabajo y unos bancos donde sentarse.

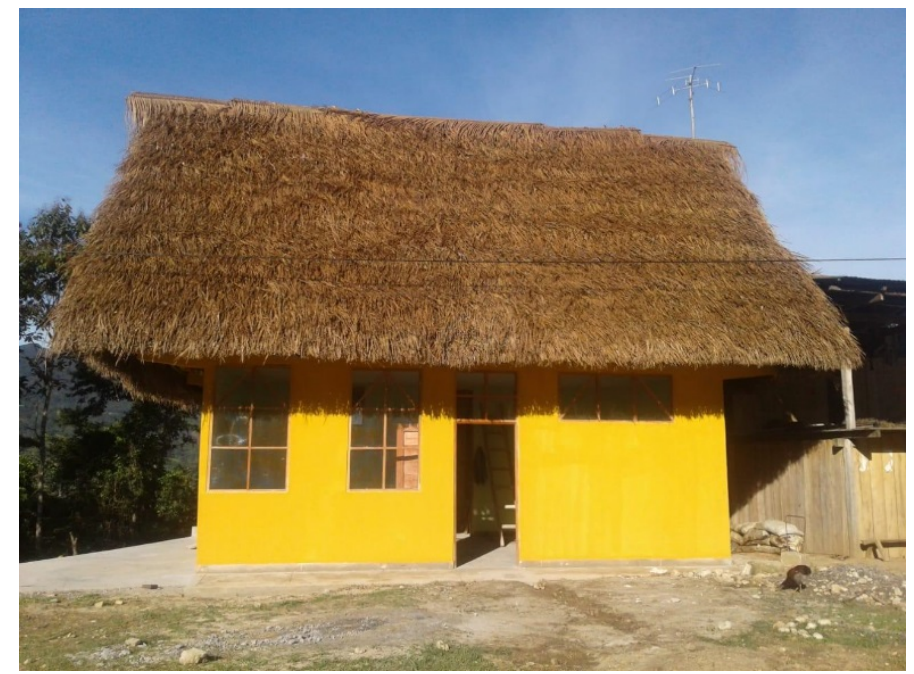

Figura 6: warmi wasy

Campo Abierto, v. 40, n. 1, p. 61-71, 2021 


\section{VALORACIÓN DE LA EXPERIENCIA}

Al finalizar la estancia en la comunidad nativa, el alumnado elaboraba una memoria en la que valoraba su experiencia y el proceso vivido. En este documento, el alumnado debía realizar un análisis de sus aportaciones a la comunidad, así como identificar los aprendizajes adquiridos durante su vivencia. A continuación, se muestran algunos fragmentos seleccionados de los escritos que las personas participantes elaboraron al finalizar su estancia en Chirikyacu.

De la primera persona que colaboró en el año 2011, recién terminadas las instalaciones de Valencia wasi, se ha extraído el siguiente párrafo.

Para conseguir la confianza de la comunidad me dedicaba a hacer visitas por las casas, conversar con mujeres y hombres, y jugar con los niños. Cuando me proponían trabajar en la "chacra" (parcelas donde siembran y trabajan casi todos los días) aceptaba, trabajaba codo con codo sacando maní y transportándolo a la comunidad a la manera nativa (cargando 5 kilos envueltos en una especie de pañuelo grande y cargados en la cabeza). Cuando alguna familia me proponía visitar a otro familiar de un pueblo cercano, para llevarles frijol y traer de vuelta fruta, me levantaba a las 5 de la mañana para acompañarlos, cargando en mi espalda los kilos que fuesen necesarios durante las diversas horas de caminata para llegar allá. Así, poco a poco, conseguí la confianza de la gente de la comunidad, a la vez que yo sentí que era mi casa. Poco a poco me di cuenta que los niños preguntaban por mí, me buscaban, ya me daban besos y se divertían jugando conmigo. Además, me sentí muy aceptada entre las mujeres, siendo una más cuando tenían conversaciones, aunque normalmente suelo ser el centro de atención. Con el tiempo, los hombres permitían que me sentara con ellos a conversar e incluso me ofrecían trago cuando llegaba la noche y se reunían, al fin y al cabo, creo que les divertía mi presencia porque suelo ser bastante bromista y entro en el juego, aunque a veces no lo entiendo todo. Aprender un poco de kechwa fue una experiencia interesante, y que permitió un poco más de acercamiento e incluso supuso un tipo de conexión entre el grupo y yo. Durante mis días de trabajo con las mujeres observé que una de ellas tenía una gran capacidad creativa y además tenía seguridad y confianza en sí misma; así pues, intenté explotar esa capacidad.

Mi presencia como extranjera sirvió para acostumbrar a los nativos a la gente que proviene de otras partes del mundo, y sobre todo que son de diferente color. Los niños ya no se asustan tanto de ver a turistas de tez blanca paseando por la comunidad, más bien les resulta curioso y les divierte su presencia. Además, se acostumbraron a confiar un poco más en la gente nueva, a abrir las puertas de su casa, aunque creo que esta cualidad ya la tenían. No obstante, ahora siento que hacia la gente extranjera son menos pudorosos. Hay que destacar, que derivado de incentivar a Luci, una mujer que resultaba la más creativa y segura de sí misma, y de hablar largos períodos de tiempo con ella, se consiguió que en la Asamblea de este pasado noviembre, donde iban a escoger a la nueva directiva de la comunidad, se propusiera como vocal y fuese aceptada por el resto de comuneros. Como desarrollo endógeno de la comunidad, esté ha sido el paso más importante que yo he vivido como una comunera más. Las cosas van cambiando, aunque poco a poco, a su ritmo.

Por otro lado, en el año 2012 una alumna del Máster de Cooperación, al solicitarle que hiciera una valoración de su estancia la tituló mi mayor aprendizaje...

En un principio las dudas asaltaban mi cabeza constantemente. ¿Qué podía hacer yo en una comunidad en medio de la selva con personas que no tienen nada que ver conmigo? ¿Realmente estoy preparada para esta experiencia? ¿He aprendido algo en el máster que pueda utilizar? Un sinfín de interrogantes que sin duda se fueron resolvieron y disipando durante mi estancia.

Fueron 5 los meses que pase trabajando codo a codo con los hombres, con las mujeres, con los niños. 5 los meses que pase aprendiendo, porque hoy sin ninguna duda puedo afirmar que he aprendido mucho más de lo que haya podido enseñar. En cada conversación, en cada taller, en cada charla, en cada caminata, en cada comida, en cada juego, en cada confidencia, en cada día a día aprendí a sacar la mejor persona que todos llevamos dentro. Aprendí a vivir sin necesidades creadas, aprendí que todas las cosas que nos unen son mucho más que las que no separan, aprendí a vivir en un contexto totalmente diferente del mío y supe que soy capaz, aprendí que la felicidad no es algo que se encuentre, sino algo que está dentro de nosotros. Hay un tipo de felicidad que ninguna cosa material puede darte, está en un paisaje, en una sonrisa, en una mirada, en un soplo de aire.

¡Me he sentido viva! La comunidad me ha dado la oportunidad de poder desarrollarme como individuo y como profesional tanto de la cooperación como de la educación, me ha permitido crear e intercambiar conocimientos. He podido poner en práctica todo lo que creía que no había aprendido en un aula. 
He aprendido que las dificultades y los errores pueden ser los mejores maestros, dificultades por tratarse de un proyecto nuevo que apenas está empezando a caminar y cuyos recursos son escasos, dificultades por ser la primera vez que se ofertan prácticas para este destino, dificultades por enfrentarme a una cultura y a un modo de proceder diferente, por sentirme sola y perdida muchas veces, por tener miedo, por no saber qué hacer ni cómo hacerlo, pero gracias a todo esto hoy puedo escribir estas líneas y afirmar que ha sido una de las mejores cosas que he hecho en mi vida.

En 2017, una alumna de Educación Social comentaba que "realizar mis prácticas profesionales en una realidad totalmente diferente, con la herramienta del diario reflexivo, se ha erigido en la metodología idónea para desarrollar mi aprendizaje en su máximo valor competencial como de capacidades, de actitudes, de valores." Esta misma alumna expresaba lo siguiente en su valoración de la estancia.

Este viaje ha supuesto en mí el despertar de los sentidos. Ahora miro al mundo con otros ojos: veo muchos más colores, sentimientos y formas, me fijo en más detalles, en las pequeñas cosas; por mi boca no salen las mismas palabras: estas están enriquecidas con condimentos como la equidad, la solidaridad, la humanidad, el respeto, el amor, la empatía...;

Que se convierta en hábito compartir todo lo que tienes, aunque tengas mucha hambre. Dejar y pedir que te inspeccionen el pelo en busca de piojos. Hervir el agua antes de tomarla, cocinar en candela, lavar la ropa a mano y la vajilla en tinas. Comer frejol, arroz y plátano cocinado de todas las formas posibles y a todas horas. Disfrutar de largas veladas con la familia Tapullima y sentirse parte. Aprender a valorar nuestras raíces, cultura y saberes ancestrales. Dejarse llevar y bailar al ritmo de nuevas melodías. Compartir apretones de manos con mujeres desconocidas, pero a la vez cercanas, en los largos viajes llenos de gente por carreteras intrincadas. No saber en qué día vives: los lunes ya no son lunes, todos los días son buenos, todos los días hay nuevas oportunidades, retos o aventuras. Razonar y cerciorarse de que tenemos que cuestionar la estructura social y el contexto y no a las personas. Emprender nuevos proyectos e ilusiones junto a la gente de aquí, trabajar juntos/as. Aprender a vivir sin necesidades creadas: entender lo bonito que es el mundo cuando estamos desconectados de las redes sociales y nos conectamos a la vida, a la naturaleza, a las verdaderas relaciones entre personas y animales.

Aprender a mirar la vida, descubrirla, sentirla y amarla. Poder desconectar de un Norte, en muchas ocasiones, avaricioso, consumista y egoísta y poder conectar con un Sur amable, natural y humano. Tejer, que va mucho más allá de confeccionar chumbes: tejer conversaciones, amistades, confianza, historias, risas, lágrimas... momentos mágicos. Cosechar café, esfuerzo y sonrisas. Pasear por las calles de Chirikyacu vistiendo una falda como la de ellas y sentirme en casa. Compartir historias, miedos, inquietudes, preguntas, curiosidades... alrededor de la candela con una taza de café caliente en la mano. Acompañar mesas pobres pero llenas de ilusión. Aprender nuevas formas, abrazarlas y amarlas: enriquecerse por lo sencillo. Aprender mucho más de lo que puedes enseñar.

Chirikyacu se ha convertido en mi hogar, mi familia, mi vida. Ha sido el pueblo humilde que me ha enseñado a caminar: la puerta que me ha permitido descubrir que otra vida es posible, otra vida muy diferente y llena de tranquilidad, paz, libertad, solidaridad y sobre todo gente buena. También la ventana que me ha permitido ver y valorar todas esas cosas tan importantes que nunca había apreciado antes.

Sin saberlo, me han regalado lo mejor que me podrían regalar.

\section{CONCLUSIONES}

Al analizar los escritos que las personas que participaron en el proyecto elaboraron al finalizar su estancia, se desprende que se ha cumplido con la esencia del Aprendizaje-Servicio ya que, por un lado, los estudiantes han aprendido a través de su participación en servicios que se realizaron en la comunidad de Chirikyacu y, por otro, la comunidad se ha visto beneficiada con las actuaciones de las y los estudiantes dirigidas a satisfacer las necesidades de la misma.

La experiencia que han tenido los participantes durante su estancia en la comunidad de Chirikyacu les ha permitido concretar con hechos prácticos los conocimientos teóricos recibidos en su formación universitaria. A modo de ejemplo, el trabajo en el huerto, más que una 
transferencia vertical de conocimientos fue un "diálogo de saberes", pues, tanto docentes como estudiantes y comuneros que participaron en el programa, compartieron y aportaron ideas, conocimiento, experiencia, etc. en cada una de las labores realizadas sobre las técnicas de producción de diferentes hortalizas y vivieron la experiencia de obtener productos hortícolas para la alimentación. De igual manera en la experiencia de la granja se intercambiaron conocimientos y se puso en práctica lo aprendido en las aulas.

Las estudiantes de ambas universidades, UV y UNSM, se involucraron en la problemática de la comunidad y aportaron soluciones con hechos prácticos que beneficiaron a niños y niñas, mujeres y población en general.

A modo de conclusión, podemos afirmar que la valoración que el alumnado ha realizado de la estancia ha sido positiva como se desprende de las opiniones que manifestaron al terminar la misma y se cumplió con los objetivos del ApS.

\section{BIBLIOGRAFÍA}

Aramburuzabala, P. Aprendizaje-Servicio: Una herramienta para educar desde y para la justicia social. Revista Internacional de Educación para la Justicia Social, 2(2), 2013, pp. 5-11,

Francisco, A. y Moliner, L. (2010). El Aprendizaje Servicio en la Universidad: una estrategia en la formación de ciudadanía crítica. Revista Electrónica Interuniversitaria de Formación del Profesorado, 13(4),6977.

Instituto Nacional de Estadística e Informática (2000). Instituto Nacional de Estadística e Informática. Lima: INEI. Recuperado de https://www.inei.gob.pe/

Puig, J. M. y Palos, J. (2006). Rasgos pedagógicos del aprendizaje-servicio. Cuadernos de Pedagogía, 357, 60-63.

i Todas las personas adultas y los padres/madres o tutores legales, en el caso de niños/as, que aparecen en las fotografías han cedido los permisos necesarios para su publicación. 